\title{
Editorial: Epigenetic Regulation of Stem Cell Plasticity in Tissue Regeneration and Disease
}

\author{
Fulvio Chiacchiera ${ }^{1 *}$, Lluis Morey ${ }^{2,3 *}$ and Chiara Mozzetta ${ }^{4 *}$ \\ ${ }^{1}$ Department of Cellular, Computational and Integrative Biology (CIBIO), University of Trento, Trento, Italy, ${ }^{2}$ Sylvester \\ Comprehensive Cancer Center, Miami, FL, United States, ${ }^{3}$ Department of Human Genetics, University of Miami Miller School \\ of Medicine, Miami, FL, United States, ${ }^{4}$ CNR -Institute of Molecular Biology and Pathology, c/o Department of Biology and \\ Biotechnology "C. Darwin”, Sapienza University, Rome, Italy
}

Keywords: stem cells, epigenetics, regeneration, cancer epigenetics, iPSC (induced pluripotent stem cell)

\section{Editorial on the Research Topic}

\section{Epigenetic Regulation of Stem Cell Plasticity in Tissue Regeneration and Disease}

\section{OPEN ACCESS}

Edited and reviewed by:

Valerie Kouskoff,

University of Manchester,

United Kingdom

${ }^{*}$ Correspondence:

Fulvio Chiacchiera

fulvio.chiacchiera@unitn.it

Lluis Morey

Imorey@med.miami.edu

Chiara Mozzetta

chiara.mozzetta@uniroma1.it

Specialty section:

This article was submitted to

Stem Cell Research,

a section of the journal

Frontiers in Cell and Developmental

Biology

Received: 09 January 2020

Accepted: 29 January 2020

Published: 19 February 2020

Citation:

Chiacchiera F, Morey L and Mozzetta C (2020) Editorial:

Epigenetic Regulation of Stem Cell Plasticity in Tissue Regeneration and Disease. Front. Cell Dev. Biol. 8:82. doi: 10.3389/fcell.2020.00082
Several adult tissues are endowed with remarkable regenerative capacities. Their architecture and functionality are preserved through the presence of a dedicated stem cell pool that fuels tissue regeneration during homeostasis or after acute injuries. Therefore, preserving tissue-specific cell identity by regulating proliferation and differentiation is key to maintain the correct function of the tissue. All the external stimuli that control tissue homeostasis and repair converge on chromatin to activate or repress specific set of genes. To coordinate the different transcriptional programs that regulate such processes, specific multiprotein complexes with specific enzymatic activities play a pivotal role. All these epigenetic activities shape the proper chromatin landscape allowing stem cell plasticity both in vivo and in vitro (Avgustinova and Benitah, 2016). The key role of the epigenetic mechanisms to preserve stem-cell identity is emphasized by their involvement in several diseases and during development. Indeed, mutations affecting the activities of chromatin modifying complexes are frequently incompatible with life or result in severe developmental defects. Several oncogenic mutations affecting chromatin modifying molecules have been also identified for being responsible for cancer initiation, progression, and invasion. Cancer cells are addicted to mutations in genes encoding epigenetic factors or to their aberrant activity when mutated (Cavalli and Heard, 2019). During the last decade, small compounds designed to target epigenetic factors to either modulate or block their activity, paved the way for the development of novel therapeutic interventions and for improved cell-based therapy (Dawson, 2017). In this Research Topic some of these aspects are presented and discussed considering the most recently published evidences.

A milestone of the last 20 years of scientific accomplishments is the possibility to modulate cell fate by reprogramming the transcriptional and epigenetic landscape of adult stem and differentiated cells. The potential to generate specific cell types capable of replacing damaged or aged human tissues is the coveted vision of regenerative medicine. Human induced pluripotent stem cells (iPSC) hold promise to fulfill this vision, as they display the potential to differentiate into any specific cellular lineage (Rowe and Daley, 2019). Clinically, this breakthrough finding is one of the most promising discoveries in regenerative medicine, opening up unprecedented opportunities for patient- specific stem cell-based therapies such as tissue replacement and drug discovery. Such a powerful approach however does not come without risks. The reprogramming process per se and the remarkable transcriptional and epigenetic changes that are induced, exposes cells to mutational events and accumulation of epigenetic abnormalities. This imposes a careful evaluation that a stable genetic and (epi)genomic state is reacquired. Perrera and Martello focus their attention on genomic 
imprinting. They thoroughly review the epigenetic alterations that could affect imprinted loci, discussing their impact on the reprogramming process, and presenting the potential hazards for clinical applications.

An extensive rewiring of the epigenetic landscape is observed during cancer formation and progression. Oncogenic mutations are frequently detected in chromatin modifying complexes, including SWI/SNF, Polycomb and COMPASS/COMPASS-Like complexes (Feinberg et al., 2016). Chan and Chen discuss the extensive rearrangements imposed to the epigenetic landscape by oncogenic MLL-fusion proteins causing leukemia. They review the roles of MLL-containing complexes during homeostasis and tumor formation and provide a compelling overview of the pharmacological strategies currently under investigation that exploit the specific vulnerabilities associated with MLL-fusion proteins-induced leukemia.

Histone- and DNA-demethylating enzymes belonging respectively to the family of the Jumonji-C domain-containing histone demethylases (JHDMs) and the ten-eleven translocation (TET) proteins represent another layer of epigenetic control and provide additional opportunities to shape chromatin landscape. While JHDMs catalyse the removal of mono-, di-, and tri- methylation marks on the lysine residues of multiple histones, TET enzymes catalyse DNA demethylation through the successive oxidation of 5-methylcytosine $(5 \mathrm{mC})$ to 5-hydroxymethylcytosine $(5 \mathrm{hmC}), 5$-formylcytosine $(5 \mathrm{fC})$, and 5-carboxylcytosine (5caC) (Schubeler, 2015). Being $\mathrm{Fe}^{2+}$ and $\alpha$-ketoglutarate-dependent dioxygenases, the activity of both these class of enzymes can be enhanced by simply using Vitamin C. Chong et al. discuss how different applications of vitamin C boost JHDMs and TETs activity. From somatic cell reprograming to the regulation of cancer cell epigenome the authors extensively reviewed Vitamin C activity, discussing new perspectives of how nutrients and metabolism can modulate chromatin state.

The role of metabolic pathways in modulating the epigenetic landscape of the cells is also the topic of the review from Purohit and Dhawan. The authors comprehensively reviewed the recent findings on the metabolic pathways sustaining muscle stem cells providing an overview of the interdependency between

\section{REFERENCES}

Avgustinova, A., and Benitah, S. A. (2016). Epigenetic control of adult stem cell function. Nat. Rev. Mol. Cell. Biol. 17, 643-658. doi: 10.1038/nrm.2016.76

Cavalli, G., and Heard, E. (2019). Advances in epigenetics link genetics to the environment and disease. Nature 571, 489-499. doi: 10.1038/s41586-019-1411-0

Dawson, M. A. (2017). The cancer epigenome: concepts, challenges, and therapeutic opportunities. Science 355, 1147-1152. doi: 10.1126/science.aam7304

Feinberg, A. P., Koldobskiy, M. A., and Gondor, A. (2016). Epigenetic modulators, modifiers and mediators in cancer aetiology and progression. Nat. Rev. Genet. 17, 284-299. doi: 10.1038/nrg. 2016.13

Rowe, R. G., and Daley, G. Q. (2019). Induced pluripotent stem cells in disease modelling and drug discovery. epigenetic modifications and metabolic reactions in preserving adult skeletal muscle homeostasis.

Altogether the reviews collected in this Research Topic provide an overview of some important epigenetic mechanisms involved in stem cell maintenance, highlighting new potential opportunities for the development of novel approaches in cancer treatments, degenerative diseases, and stem cell-based applications. Pharmacological manipulation of the epigenetic landscape is now one of the most attractive and intense field of investigation. Several compounds, the so-called "epi-drugs," are currently tested in clinical trials as potential treatments in different cancer types. Of note, a number of previously developed compounds have been shown effective in reverting aberrant disease-associated epigenetic states, indicating that drug repurposing might likely accelerate their transition toward clinical application.

\section{AUTHOR CONTRIBUTIONS}

All authors listed have made a substantial, direct and intellectual contribution to the work, and approved it for publication.

\section{FUNDING}

This work in Fulvio Chiacchiera Laboratory was supported by grants from the Italian Association for Cancer Research (AIRC, MFAG 2017 - ID 20344). Lluis Morey Laboratory was funded by Comprehensive Cancer Center funds, AACRBayer Innovation and Discovery grant (18-80-44-MORE), Flight Attendant Medical Research Institute (FAMRI) Breast Cancer Developmental Grant, ACS-American Cancer Society (IRG-17183-16), Stanley J. Glaser Foundation Research Award (UM-SJG2020-3), Leukemia and Lymphoma Society Specialized Center of Research Grant (LLS-SCOR), and the Lampert Breast Cancer Research Fund. Chiara Mozzetta Laboratory was funded by Italian Ministry of University and Research (SIR, Scientific Independence of Young Researcher no. RBSI14QMG0), Italian Association for Cancer Research (AIRC; MFAG no. 18993), AFM-Telethon (\#22489), and the CNCCS (Collection of National Chemical Compounds and Screening Center), LIFE2020Regione Lazio.

\section{Nat. Rev. Genet. 20, 377-388. doi: 10.1038/s41576-019- 0100-z}

Schubeler, D. (2015). Function and information content of DNA methylation. Nature 517, 321-326. doi: 10.1038/nature14192

Conflict of Interest: The authors declare that the research was conducted in the absence of any commercial or financial relationships that could be construed as a potential conflict of interest.

Copyright $\odot 2020$ Chiacchiera, Morey and Mozzetta. This is an open-access article distributed under the terms of the Creative Commons Attribution License (CC BY). The use, distribution or reproduction in other forums is permitted, provided the original author(s) and the copyright owner(s) are credited and that the original publication in this journal is cited, in accordance with accepted academic practice. No use, distribution or reproduction is permitted which does not comply with these terms. 